\title{
Barrett's esophagus: endoscopic treatments I
}

\author{
Michael D. Saunders, ${ }^{1}$ Alejandro Nieponice, ${ }^{2,3}$ Katerina Dvorak, ${ }^{4}$ Aaron Goldman, ${ }^{4}$ \\ Edgardo Diaz-Cervantes, ${ }^{5}$ A. De-la-Torre-Bravo, ${ }^{6} \mathrm{~S}$. Sobrino-Cossio, ${ }^{7}$ E. Torres-Durazo, ${ }^{8}$ \\ O. Martínez-Carrillo, ${ }^{5} \mathrm{~J}$. Gamboa-Robles, ${ }^{9}$ Melissa Upton, ${ }^{10}$ Henry D. Appelman, ${ }^{11}$ \\ Luigi Bonavina, ${ }^{12}$ Richard I. Rothstein, ${ }^{13}$ and Vic Velanovich ${ }^{14}$ \\ ${ }^{1}$ Digestive Disease Center, University of Washington Medical Center, Seattle, Washington. ${ }^{2}$ University of Pittsburgh, \\ Pittsburgh, Pennsylvania PA. ${ }^{3}$ University of Favaloro, Buenos Aires, Argentina. ${ }^{4}$ Department of Cell Biology and Anatomy, \\ College of Medicine and Arizona Cancer Center, The University of Arizona, Tucson, Arizona. ${ }^{5}$ Saint Joseph's Hospital, \\ Gastroenterology, Endoscopy and Motility Unit, Zacatecas, México. ${ }^{6}$ Angeles Metropolitan Hospital, Mexico, Distrito Federal, \\ México. ${ }^{7}$ Cancerology's National Institute, México, Distrito Federal, México. ${ }^{8}$ Angeles Mocel Hospital, Siglo XXI, México, \\ Distrito Federal, México. ${ }^{9}$ General Hospital, Pathology Division, Fresnillo Zacatecas, México. ${ }^{10}$ University of Washington \\ Medical Center, Seattle, Washington. ${ }^{11}$ Department of Pathology, The University of Michigan Hospitals, Ann Arbor, Michigan \\ MI. ${ }^{12}$ Department of Surgery, IRCCS Policlinico San Donato, University of Milano Medical School, Milan, Italy. ${ }^{13}$ Section of \\ Gastroenterology, Dartmouth-Hitchcook Medical Center, Lebanon, New Hampshire. ${ }^{14}$ Division of General Surgery, Henry Ford \\ Hospital, Detroit, Michigan
}

The following on endoscopic treatments of Barrett's esophagus includes commentaries on indications for endoscopic treatments; endo-luminal plication procedures; the cellular modifications induced by the endoscopic ablation therapies; eradication by banding without resection; the evaluation of complete ablation; recurrence after ablation; association of antireflux surgery; radiofrequency ablation; and nondysplastic Barrett's esophagus.

Keywords: Barrett's esophagus; esophagectomy; esophageal cancer; radiofrequency ablation; dysplasia; goblet cell; band ligation; banding without-resection; banding without-snare; fundoplication; GERD; antireflux surgery; endolumenal procedures; intramucosal carcinoma

\section{Concise summaries}

- There is good evidence supporting the efficacy and safety of endoscopic treatment (photodynamic therapy (PDT), RFA, endoscopic resection (ER)) in early Barrett's cancer. The role of endoscopic treatment for those with no dysplasia or low-grade dysplasia (LGD) is uncertain and cannot be uniformly recommended at this time.

- The current optimal approach for endoscopic management of early Barrett's cancer is ER of visible mucosal lesions followed by eradication of any residual Barrett's mucosa with RFA.

- It is not clear if ablative therapies reduce cancer risk. In susceptible BE patients, they may induce formation of cells with damaged DNA and mutations and thus with higher risk to develop esophageal adenocarcinoma (EAC). Additional markers that have been reported to be abnormal in Barrett's intestinal metaplasia and EAC deserve to be studied.

- The most recent devices launched in the field of endoluminal plication procedures allow for the creation of a valve that resembles a laparoscopic fundoplication with a high-pressure zone and interesting anatomical appearance.

- Argon plasma coagulation (APC), at a power output of 60-90 W, allows a safe ablation of $\mathrm{BE}$ and LGD with a high success rate. In most series of patients treated with APC, the newly developed squamous epithelium remains stable at up to five-year follow-up.

- Radiofrequency ablation (RFA) seems optimal for treating flat, nonnodular Barrett's esophagus (BE). Nodular disease may be treated with RFA following the removal of the nodular segments with endoscopic mucosal resection. Proof of true cancer reduction with implementation of 
RFA as a treatment strategy in nondysplastic BE is still lacking.

- Banding without-resection (BWR) is a new ablation modality that probably collects the desired criteria for a simple and effective ablative technique for BE. BWR has some advantages and may have fewer risks than EMR and other ablative modalities because it is minimally invasive.
- The factors of recurrence of Barrett's mucosa after ablation cannot be defined yet, but the difference between recurrence and hidden residual Barrett's mucosa has to be defined first.

- It has been shown that the addition of an antireflux operation, most typically a laparoscopic Nissen fundoplication, reduces the rate of recurrent or persistent BE.

\section{What are the current indications and results of the endoscopic treatments of BE?}

Michael D. Saunders

mds@u.washington.edu

The role of endoscopic therapy in BE has evolved. Once considered experimental for high-grade dysplasia (HGD), it is now becoming established as the treatment of choice in that setting. The indications for endoscopic therapy in BE are summarized in Table 1.

Endoscopic therapies available include ER, RFA, photodynamic therapy (PDT), cryotherapy, and other modalities such as APC, multipolar electrocautery, and laser therapy. There is good evidence supporting the efficacy and safety of endoscopic

Table 1. Indications for endoscopic therapy in Barrett's esophagus

\begin{tabular}{|c|c|}
\hline Pathology & $\begin{array}{l}\text { Endoscopic treatment } \\
\text { recommended? }\end{array}$ \\
\hline $\begin{array}{l}\text { HGD, intramucosal } \\
\text { carcinoma (early } \\
\text { Barrett's cancer) }\end{array}$ & Definitely \\
\hline \multirow[t]{2}{*}{$\begin{array}{l}\text { Nondysplastic Barrett's, } \\
\text { indefinite, low-grade } \\
\text { dysplasia }\end{array}$} & Not uniformly \\
\hline & $\begin{array}{l}\text { Consider if other factors } \\
\text { present such as family } \\
\text { history of esophageal } \\
\text { cancer }\end{array}$ \\
\hline \multirow[t]{2}{*}{$\begin{array}{l}\text { Localized, invasive cancer } \\
\text { in nonsurgical } \\
\text { candidate }\end{array}$} & Individualized \\
\hline & Considered palliative \\
\hline
\end{tabular}

treatment (PDT, RFA, and ER) in early Barrett's cancer. The role of endoscopic treatment for those with no dysplasia or LGD is uncertain and cannot be uniformly recommended at this time.

\section{Endoscopic resection}

ER is superior to mucosal biopsies for diagnosis and staging of dysplasia, changing the initial histologic diagnosis and management in about $25 \%$ of cases. ${ }^{1}$ ER is an excisional therapy, allowing for removal of visible neoplastic mucosal lesions. Negative margins obtained with ER correlate with surgical pathology. Importantly, there is a low rate $(<3 \%)$ of lymph node metastases with mucosal neoplasia (intramucosal carcinoma). ${ }^{2}$ Following ER, metachronous lesions develop in approximately 20\% during follow-up. ${ }^{3}$

\section{Ablation}

The only modalities studied in controlled fashion for early Barrett's cancer are PDT and RFA (Table 2). Both were superior to medical therapy in eradication of HGD and decreased progression to cancer. Stricture rate was higher with PDT than RFA.

\section{Multimodality therapy}

The current optimal approach for endoscopic management of early Barrett's cancer is ER of visible mucosal lesions followed by eradication of any residual Barrett's mucosa, with RFA being the ablative procedure of choice. Table 3 below summarizes the prospective studies evaluating multimodality therapy, highlighting an efficacy of over $90 \%$ in eradication of dysplasia and Barrett's mucosa with relatively low complication rates (13-17\%).

\section{Recommendations}

Endoscopically visible mucosal lesions are removed with ER, allowing for accurate staging and potentially curative excision of the mucosal neoplasia. ER 
Table 2. Summary results for endoluminal plication devices

\begin{tabular}{lll}
\hline & \multicolumn{1}{c}{ PDT $(n=138)$} & \multicolumn{1}{c}{ RFA $(n=42)$} \\
\hline Author & Overholt et al. ${ }^{5}$ & Shaheen et al. $^{6}$ \\
Medical therapy & Omeprazole $20 \mathrm{mg} \mathrm{BID}$ & Esomeprazole 40 mg BID \\
Nodular disease & $33 \%$, additional $50 \mathrm{~J} / \mathrm{cm}$ PDT & $9 \%$, EMR allowed prior to study entry \\
Ablation treatments & Mean 2.3 & Mean 3.5 \\
Follow-up & 24 months & 12 months \\
Eradication of IM & $52 \%$ vs. $7 \%$ & $74 \%$ vs. $2.3 \%$ \\
Eradication of HGD & $77 \%$ vs. $39 \%$ & $81 \%$ vs. $19 \%$ \\
Progression to cancer & $13 \%$ vs. $28 \%$ & $2.4 \%$ vs. $19 \%$ \\
Stricture & $36 \%$ & $6 \%$ \\
\hline
\end{tabular}

Adapted from Wolfsen. ${ }^{4}$

Table 3. Diseases with cancer-prone epithelium and operations designed to reduce cancer risk

\begin{tabular}{lccccc}
\hline Study & $n$ & Modality & Outcome & Follow up Complications \\
\hline Pech et al. ${ }^{3}$ & 349 & EMR (255) EMR + PDT (11) & $\begin{array}{l}\text { Long-term complete response 95\% } \\
\text { 5-year survival 84\% }\end{array}$ & 64 months & $17 \%$ \\
Pouw et al. & CR-N 100\%, CR-IM 93\% & EMR + RFA & CR-N & 12 months & $13 \%$ \\
\hline
\end{tabular}

${ }^{a}$ Euro II study: interim analysis of ongoing prospective multicenter trial.

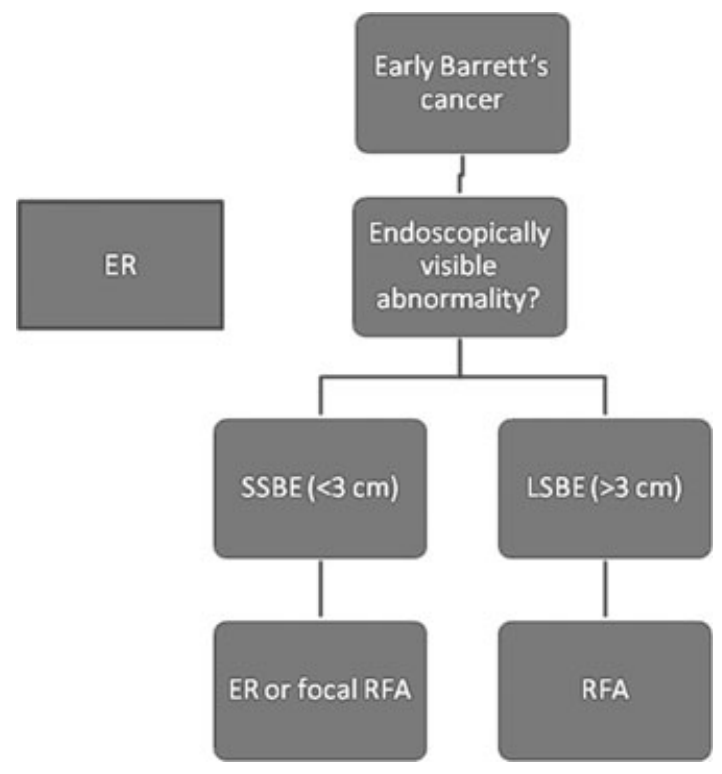

Figure 1. Current optimal approach to endoscopic treatment of early Barrett's cancer.

can be safely performed to semi-circumferential, $2 \mathrm{~cm}$ in length areas. ER is repeated until all endoscopically visible abnormalities are removed. The residual Barrett's mucosa should then be eradi- cated given the risk of developing metachronous lesions.

RFA is the preferred ablation modality in BE following ER of visible mucosal lesions or in flat dysplasia. For long segments of Barrett's mucosa $(>3 \mathrm{~cm}$ ), balloon-based RFA (Halo 360) is preferred initially. For short segments or residual Barrett's following balloon ablation, probe-based RFA (Halo 90) is recommended. Alternatively, ER can be performed exclusively in those with short segments of Barrett's $(<3 \mathrm{~cm})$. The recommended approach to endoscopic treatment of early Barrett's cancer is summarized in the following algorithm (Fig. 1).

\section{What are the results of the endoluminal plication procedures?}

Alejandro Nieponice

Nieponicea@upmc.edu

In the last decade, several endoluminal devices have gained attention as promising tools for treatment of gastresophageal reflux disease (GERD) as an alternative to laparoscopic fundoplication. However, several requirements need to be met for them to prove successful: they need to be safe, effective, 
Table 4. Summary results for endoluminal plication devices

\begin{tabular}{|c|c|c|c|c|c|c|}
\hline \multirow[t]{4}{*}{ Plicator (NDO) } & Rothstein & 2006 & 78 & $3 \mathrm{~m}$ & 50 & 23 Plicator \\
\hline & & & 80 & & 24 & 15 sham \\
\hline & Pleskow & 2007 & 29 & 3 yrs & 57 & $\mathrm{n} / \mathrm{a}$ \\
\hline & & 2008 & 33 & 5 yrs & 67 & $\mathrm{n} / \mathrm{a}$ \\
\hline \multirow[t]{2}{*}{ EndoCinch (Bard) } & Abou-Rebyeh & 2005 & 38 & $1 \mathrm{yr}$ & 20 & 14 \\
\hline & Schiefke & 2005 & 70 & $1.5 \mathrm{yrs}$ & 6 & 28 \\
\hline \multirow[t]{4}{*}{ EsophyX (EGS) } & Cadière - Phase 1 & 2008 & 17 & $1 \mathrm{yr}$ & 82 & 63 \\
\hline & & 2009 & 14 & $2 \mathrm{yrs}$ & 79 & $\mathrm{n} / \mathrm{a}$ \\
\hline & Cadière - Phase 2 & 2008 & 79 & $1 \mathrm{yr}$ & 85 & $37 / 48$ \\
\hline & & 2009 & 51 & $2 \mathrm{yr}$ & 71 & $\mathrm{n} / \mathrm{a}$ \\
\hline
\end{tabular}

user-independent, and economically viable. That strong combination has yet to be seen and has driven several of these devices to failure. As opposed to bulking agents, plication devices have made further achievements by starting with the right concept of recreating the anatomy of a valve at the GE junction. But this concept is far from being a simple task and technical challenges have hampered most of the attempts. The first plication device was Endocinch (BARD, Inc., Murray Hill, NJ, USA), which showed good initial control of symptoms but lack of objective reflux control with $\mathrm{PH}$ monitoring. The symptom control failed in the long-term follow-up, plication seemed to be loosened, and this procedure has been abandoned. ${ }^{8}$ With similar results but a mild improvement in reflux control the Plicator (NDO Surgical, Inc., Mansfield, MA, USA) has shown a better transmural fixation trying to ensure serosa-to-serosa contact. ${ }^{9}$ However, a real valve with a long esophageal segment could not be achieved. This procedure has also been discontinued by most of the surgical community. The most recent device launched to the market is the Esophyx (EndoGastric Solutions) that allows the creation of a valve that resembles a laparoscopic fundoplication with a high-pressure zone and interesting anatomical appearance. Initially reported results were more encouraging than previous competitors and mixed results started to appear as the technique spreads out. $^{10,11}$

A large clinical trial undergoing in the U.S. will probably set faith for this approach. A summary of available results for all these devices is reported in Table 4 . In order to accurately understand the results of the endoluminal plication procedures, we need to analyze what the expectations were, what we compared them to, and what the optimal patient population is. Initially the stakes were set too high as everyone thought of this as the perfect alternative to withdraw patients from PPIs without the need of undergoing a laporoscopic surgery and its known side-effects. Comparison with laparoscopic surgery is probably the wrong idea for these devices, as we have learned that hiatal hernias are a big limitation for these procedures. If there is a room for an endoluminal procedure, it will be in a middle-world between chronic medication and the Nissen fundoplication. Therefore, comparisons should be made with medical therapy.

And last but definitely not least, patient selection will turn out to be the key if any endoluminal plication will survive. In a retrospective study, Khajanchee et al. showed that patients with lower DeMeester scores, heartburn scores lower than 2, and body mass index lower than 30 had a significantly better outcome when undergoing an endoluminal plication.

This tendency shows that only a highly selected population will benefit from these technologies making the market still available but much less attractive for the companies that need to work on the evolution of these devices. This fact deserves a paragraph on insurance carriers that are ever searching for ways to reduce medical costs, and they are very cautious after the first iteration of endolumenal antireflux procedure and require concrete evidence of efficacy prior to authorizing payment for these novel procedures. George Bernard Shaw said "When I was a young man I observed that nine out of ten things I did were failures. I didn't want to be a failure, so I did ten times more work". This seems to be the case if we want to find a safe, effective and reliable endoscopic method for our patients. 


\section{May endoscopic ablation therapies induce formation of cells more susceptible to genomic damage?}

\section{Katerina Dvorak and Aaron Goldman kdvorak@email.arizona.edu}

$\mathrm{BE}$ is a premalignant condition associated with the development of EAC. Patients with this cancer have a poor prognosis with a median survival of less than one year. It is becoming clear that acid suppression therapies alone, using surgery or drugs, have no significant effect on reversing BE. Endoscopic ablation therapies are becoming more popular in recent years, especially for patients with HGD or with intramucosal adenocarcinoma. Endoscopic ablation of abnormal tissue is less invasive and safer compared to esophagectomy that used to be the only routine clinical treatment of HGD and EAC. A number of endoscopic therapies to eradicate esophageal columnar epithelium were developed over recent years including APC, multipolar electrocoagulation, $\mathrm{PDT}$, laser therapy, cryotherapy, and RFA. However, concerns remain regarding incomplete ablation, strictures, and buried glands that are not readily detected and may represent a risk for esophageal cancer development.

In the setting of complete ablation, the neosquamous epithelium is not different from normal squamous epithelium and represents low cancer risk. Garewal et al. have shown low expression of Ki67, p53, and low activities of ornithine decarboxylase in new squamous epithelium in completely ablated esophagus. ${ }^{12}$ Furthermore, new squamous epithelium exhibits low level of staining for Ki67 and p53 as well as no DNA abnormalities after RFA. Cytokeratin expression (CK-8 and-14) and miRNAs were also similar in postablation neosquamous epithelium and normal squamous epithelium.

However, in the setting of incomplete ablation the data are not so clear. Krishnadath et al. reported that three patients who were free of dysplasia after photodynamic therapy developed dysplasia during followup and also had increased expression of at least one of the markers associated with increased cancer risk (Ki67, aneuploidy, p53 expression, or p16 promoter hypermethylation). ${ }^{13}$ Similarly, Hage et al. found that after ablation therapy, residual or recurrent $\mathrm{BE}$ glands retain or accumulate abnormalities such as p53 overexpression, increased proliferation, and DNA abnormalities even in the absence of dyspla- sia. ${ }^{14}$ In contrast, a recent biomarker study by Prasad et al. shows that DNA abnormalities detected by fluorescence in situ hybridization (FISH) were significantly decreased after PTD therapy. However, a subset of patients (19\%) without dysplasia had positive FISH results and developed recurrent HGD. ${ }^{15}$ Hornick et al. reported that buried glands after PDT show reduced crypt proliferation and lower level of DNA abnormalities, and they suggest that these glands have lower neoplastic potential.

The majority of these studies evaluated only biopsies of columnar epithelium from incompletely ablated patients. Only few studies evaluated changes in squamous epithelium after ablative therapies and only few patients were included in these studies. Furthermore, even fewer studies were focused on biomarker alterations at new squamo-columnar junctions that are formed after incomplete ablation. Our group has shown that these new junctions stain positively for several cancer risk associated biomarkers, such as cyclooxygenase 2, Ki67, and p53 in a subset of patients. ${ }^{16}$ Importantly, the expression of these biomarkers was low in the preablation BE biopsies.

The major approach to eradicate $\mathrm{BE}$ is combination of injury by different ablative techniques and acid suppression. In this environment, intestinal epithelium is healed and replaced primarily with neosquamous epithelium; however, the effect on cancer risk remains unknown, and the question of whether ablation decreases the cancer risk remains unanswered. Wound healing that follows ablation is a complex process that involves different cytokines, enzymes and growth factors, and activation of signaling pathways. This process can be divided into inflammation, proliferation, and maturation phases. After injury, inflammatory cells invade the wound tissue. They produce proteinases, reactive oxygen species, and reactive nitrogen species as a defense against bacteria. Inflammatory cells are also an important source of growth factors and cytokines, which initiate the proliferative phase of wound repair. Wound healing is associated with increased expression of IL- $1 \alpha$, IL-1 $\beta$, IL- 6 , TNF- $\alpha$, or hepatocyte growth factor (HGF) and members of the epidermal growth factor (EFG) family. Importantly, overexpression of these cytokines and growth factors and their receptors is often found in $\mathrm{BE}$ and $\mathrm{EAC}$ and is likely to have a role in esophageal tumorigenesis. Furthermore, reactive oxygen species produced by inflammatory cells can cause DNA damage 
resulting in mutations. Most of the genes that orchestrate the wound-healing process are also important regulators of cancer growth and progression. Therefore, epithelium undergoing these changes is possibly more prone to genomic instability and is susceptible to DNA damage in a subset of BE patients especially those, who require repeated ablation. In addition, DNA damage and increased cytokine and growth factor expression may even be elevated if acid and bile reflux is not well controlled. Indeed, both these components of refluxate were shown to induce DNA damage.

In summary, it is not clear if ablative therapies reduce cancer risk. In susceptible $\mathrm{BE}$ patients, they may induce formation of cells with damaged DNA and mutations and, thus, with higher risk to develop EAC. Additional markers that have been reported to be abnormal in Barrett's intestinal metaplasia and EAC deserve to be studied to further address this important clinical issue.

\section{What can be expected from Banding without-resection for Barrett's esophagus?}

Edgardo Diaz-Cervantes

edgardoscopio@hotmail.com

Most of the ablative treatments for Barrett's esophagus are expensive, potentially hazardous, require specialized training, and are not universally available. Furthermore, these ablative techniques may fail to eradicate all of the Barrett's epithelium and often leave remnants of metaplastic mucosa behind in the form of visible islands or in the form of glands buried under a layer of neosquamous epithelium. ${ }^{17}$ An effective ablative therapy for BE that is relatively inexpensive, safe, simple, and readily available would be highly desirable. We have been working on BWR from April 2004 to date (seven years); this work has been formerly published in $2007 .{ }^{18}$

BWR is a new ablation modality that probably collects the above desired criteria for an ideal ablative technique. It is simple, because there is no need for special skills-you just need an endoscope, a multiband ligator, and some technique; there is no need for an injector, snare, or anything else. BWR is also very effective: during the last seven years we have gotten rid of Barrett's in 110 patients, by banding one or two spots of Barrett's epithelium on each session and repeating at three month intervals until the metaplastic epithelium has been completely eradicated. We have obtained $98 \%$ of complete resolution of intestinal metaplasia compared to only $2 \%$ in our control group consisting of 76 patients (with omeprazol bid for years, only). BWR is a minimally invasive and "easy" procedure for the esophagus because only a very small, superficial

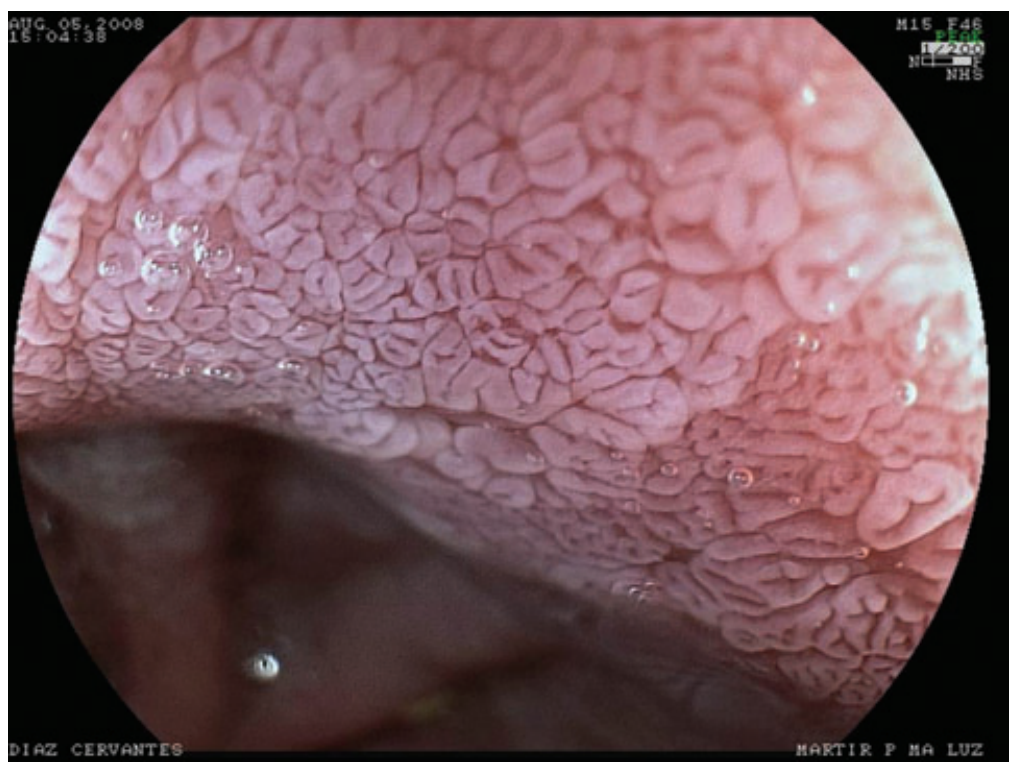

Figure 2. This C1M3 BE was completely ablated in just 2 sessions and 3 rubber bands. The image is enhanced by magnification and acetic acid. 
ulceration must heal-the residual scar is thin, small, superficial, and this is probably the main reason for the absence of residual stenosis. BWR is not expensive and is available worldwide. This new Barrett's ablation technique is "low tech" but has "high results."

\section{Number of banding and sessions for complete resolution of $B E$}

How many banding sessions were needed to get complete resolution of $\mathrm{BE}$ in the treated group consisting of 101 SSBE and 9 LSBE segment Barrett's? A mean of 2.6 sessions (very similar to other ablation procedures). How many bands were needed for complete resolution of $\mathrm{BE}$ in these patients? A mean of 4.7 bands.

\section{Long-lasting changes}

One of the big concerns after any ablation procedure is what happens years after ablation and how durable the changes are. In our 110 ablated patients, posteradication changes seems to be durable. The longer follow up in 44 of our 110 patients ranges between 50 and $70 \mathrm{~ms}$ with permanent or durable changes. After the last banding procedures, there is no modification at all, on the endoscopic finding even after four to six years (Fig. 3).

\section{Comparison between techniques}

A frequent inquiry about BWR is the lack of tissue sampling after ablation; the answer to this is that ablation modalities do not enable tissue sampling after ablation because, except for endoscopicmucosal resection, all of them are in situ tissue-destroying techniques. BWR has a deeper penetration mechanism, the same as endoscopic mucosal resection EMR (submucosal), whereas other ablation procedures affect only the mucosal layer. Because of its mechanism of action, this nonresection technique avoids the risk of perforation and bleeding (Fig. 2).

\section{Conclusions}

(1) BWR is a new ablation procedure for Barrett's and is very different than EMR.

(2) BWR has the potential to remove HGD because it achieves good depth of ablation.

(3) BWR has many advantages and appears to have fewer risks than EMR and other ablative modalities because it is minimally invasive ("easy" on the esophagus).

(4) In our 110 patients, we found no complications at all (no stricture, bleeding, or perforation), and $98 \%$ complete resolution of intestinal metaplasia.

(5) BWR may be the procedure of choice in cirrhotic patients with $\mathrm{BE}$ and $\mathrm{HGD}$

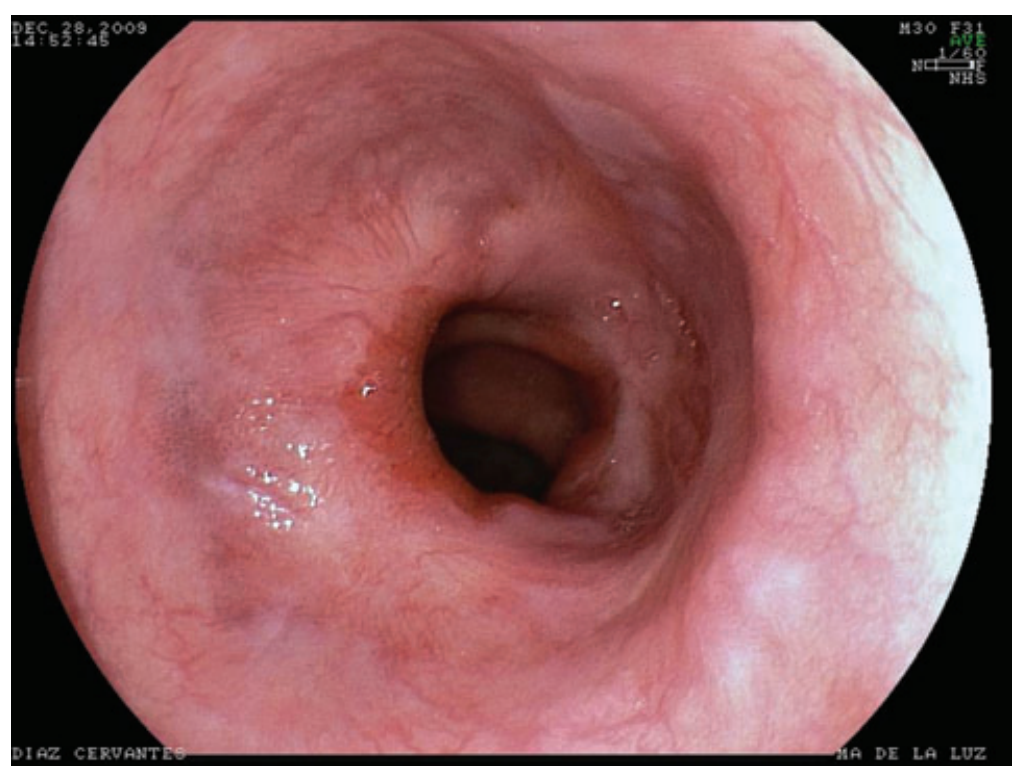

Figure 3. The same patient as shown in Fig. 2 one year after 2 banding sessions and 3 rubber bands. There is no stenosis at all. Four years of follow up has demonstrated no further changes, with apparent BE endoscopically and histologically cleared. 
(6) Posteradication changes seem to be durable even after six years of follow-up.

BWR appears to be a safe, effective, simple, and widely available technique for eradicating shortsegment and tongued large-segment BE. Nevertheless, further studies are needed.

\section{How should complete ablation of Barrett's mucosa be defined?}

\author{
Melissa P. Upton \\ mupton@u.washington.edu
}

Before defining "complete ablation of Barrett mucosa," it is essential to acknowledge that there is still no international agreement regarding the definition of Barrett mucosa. What are we trying to ablate?

The original definition by Mr. Norman Barrett was columnar lining of the tubular esophagus, and it dominated the thinking of physicians and scientists for decades. It remains the definition in England and in some other countries, including Japan. According to this definition, esophageal columnar lining can include any type of columnar metaplasia-oxyntic, cardiac, and intestinalized mucosae. This would also include multilayered epithelium, a hybrid epithelium identified more recently and seen in association with chronic reflux. Using this definition, complete ablation would require ablation of all columnar lining in the esophagus.

In contrast, the American definition of Barrett's esophagus, adopted in the mid-1990s, requires an endoscopic-histologic correlation. The endoscopist must recognize irregular tongues of pink columnar-appearing epithelium extending into the esophagus proximal to the GE junction, and the pathologist must confirm the histologic finding of specialized columnar epithelium, intestinalized epithelium with goblet cells containing acidic mucins, in biopsies designated as esophageal in location. The American definition came into use following the recognition that the overwhelming majority of cases of EAC and high-grade glandular dysplasia occurred in a setting of columnar metaplasia with goblet cells detected on biopsy. Using the American definition, if the goal is ablation of all Barrett mucosa, we would theoretically ablate the columnar lining of the esophagus only in patients with histologically documented goblet cells.
One problematic issue with the American definition is the challenge of sampling, because goblet cell distribution may be extensive, or focal and patchy. Occasionally, many biopsies may be needed to identify goblet cells, especially in patients with extensive neoplastic alterations. Although the Seattle protocol of biopsy sampling has been recommended as the standard approach, it is clear from a review of practice settings that many gastroenterologists do not use large-jaw biopsy forceps or take the recommended numbers of biopsies at the prescribed distance intervals between biopsies outlined in this protocol. In addition, both in historic mapping series and in more recent studies of endoscopic mucosal resection specimens, cases of adenocarcinoma have been reported in a setting of columnar lined esophagus without histologic documentation of goblet cells. The epithelium adjacent to and in carcinomas may not have identifiable intestinal phenotype. In addition, aneuploidy, and cell cycle abnormalities have been reported in epithelium without histologic features of neoplasia.

It is likely that intestinal metaplasia is simply a histologic marker of risk. Neoplastic changes probably arise in a mutated stem cell population with the capability of differentiating into a neoplastic clone with or without histologically recognizable intestinal phenotype or acidic mucin production. In addition, there may be important contributions to the development of neoplastic risk by stromal components and by populations of the submucosal glands of the esophagus. ${ }^{19}$ Ablation limited to the mucosa or superficial epithelium and lamina propria may not be adequate. To confirm if endoscopic ablative techniques can eradicate the risk of future neoplasia, will require longer follow-up intervals, rigorous and careful postablation surveillance biopsies, and additional investigation into the molecular mechanisms of neoplastic development. More work is needed to identify the cells of origin and the permissive microenvironment that favors neoplasia.

In addressing the issue of complete ablation, what are we trying to ablate? Is the goal to ablate neoplastic mucosa? Presumably, the goal is not to simply ablate already detectable neoplastic epithelium, but also to ablate mucosa that is at risk for future neoplastic alterations. Will we refer only patients with documented goblet cells, associated with higher risk for malignancy in the American series? Will we include patients with any columnar 
lining of the esophagus, given recent EMR data suggesting that neoplasia may develop in cardiac mucosal background?

Some investigators have suggested that all patients with Barrett's esophagus are potentially at risk for neoplasia and should be considered for ablation of the entire columnar lining. But ablation is costly, and patients will require continued surveillance after ablative treatment. Therefore, any decision for mucosal ablation must balance the potential costs and risks of the procedure and continued surveillance with the benefits of ablation. Instead of ablating all patients with Barrett's mucosa, perhaps we should treat only selected groups within the large population of patients with $\mathrm{BE}$, treating only those with histologically proven neoplasia or those who show specific molecular markers for increased risk of progression, such as aneuploidy or increased synthetic fraction when studied by DNA flow cytometry.

Future studies are needed to address whether molecular markers, such as methylation or mutation of p16, can assist in selection of patients at increased risk for neoplasia for whom ablation is clearly indicated. And we must design robust and thorough methods of follow-up for treated patients, both to avoid missing neoplasms that may develop in the postablation period, and also to gain understanding of how the esophagus and its microenvironment (including molecular events) are altered by ablation procedures, and if this alters the risk for future neoplastic changes.

\section{Can the factors of recurrence of intestinal metaplasia (Barrett's mucosa) be defined?}

Henry D. Appelman
appelman@umich.edu

This can be paraphrased to this question: once Barrett's mucosa is destroyed by any type of ablation, and it recurs, is there an explanation for the recurrence? To answer this question, we need data from a study that begins with Barrett's mucosa, which is then ablated by any technique and replaced by new squamous epithelium. Then the study design has to find a way to prove unequivocally that the Barrett's has been completed destroyed, and that no residual Barrett's mucosa is present beneath the new squamous mucosa, presumably by biopsy of the entire previous Barrett's segment. The study then must continue with extensive follow-up, and in those cases in which the Barrett's mucosa recurs, the study must identify the reasons for the recurrence. I looked everywhere for this study, and I could not find it. So I looked for published hints.

In one study of 72 patients with $2-6 \mathrm{~cm}$ of Barrett's mucosa treated with radio frequency ablation, $92 \%$ achieved complete response with no recurrence at five years. ${ }^{20,21}$ The definition for complete response was the lack of residual intestinal metaplasia in any biopsy. The biopsy protocol involved four quadrants at every level in one to two centimeter increments, which clearly left a lot of esophageal mucosa not sampled. $8 \%$ of these patients did not have a complete response, possibly indicating recurrence in some patients. However, no factors were evaluated in this $8 \%$ to help me answer the question above.

In another study on laser ablation in 31 patients, 21 achieved complete response, which was not clearly defined in the study, but presumably it was lack of intestinal metaplasia on any biopsy. ${ }^{22}$ Unfortunately, the biopsy protocol was not defined. Eight patients had recurrence of Barrett's from 6 to 44 months after presumably successful ablation, and six of these had a colonic epithelial protein antigen in cardiac-type epithelium without goblet cells, the Das antigen suggesting that this protein is a reasonable predictor of recurrence. However, the antigen was expressed in cardiac-type mucosa, often buried under squamous mucosa, not in goblet cell-containing mucosa. In the British definition of Barrett's mucosa, this cardiac-type mucosa with the Das antigen would have been considered Barrett's mucosa if there were appropriate endoscopic findings.

One likely explanation for recurrent Barrett's mucosa is that this mucosa has become covered by metaplastic squamous epithelium induced by the ablation technique, and this hid residual Barrett's from endoscopic detection and from biopsy. No study can guarantee that this has not happened, because no study followed ablation with biopsy of the entire previous Barrett's segment. Another explanation is that Barrett's recurred, because the new squamous epithelium induced by ablation is subject to the same factors that caused the Barrett's mucosa to occur originally. If we only knew how Barrett's 
developed in the first place. Unfortunately, we do not.

To summarize, can the factors of recurrence of Barrett's mucosa after ablation be defined? The answer is not yet, but we should keep trying. However, first we have to define the difference between recurrence and hidden residual Barrett's mucosa.

\section{Is the combination of APC with antireflux surgery a good means to enhance late results?}

\section{Luigi Bonavina}

luigi.bonavina@unimi.it

$\mathrm{BE}$ is defined as a biopsy-proven change in the distal esophageal epithelium, with normal squamous epithelium being replaced by columnar epithelium containing goblet cells. This condition, also known as intestinal metaplasia, occurs in approximately $10 \%$ of patients with gastroesophageal reflux disease. ${ }^{23}$ Intestinal metaplasia is a premalignant condition associated with an increased risk of EAC especially when LGD or HGD is found in the biopsies. On the other hand, molecular alterations may even precede the development of intestinal metaplasia in BE. $^{24}$

Retrospective studies have demonstrated that patient survival is improved if an endoscopic surveillance strategy detects cancer at earlier stages compared to a strategy of no surveillance. ${ }^{25}$ However, surveillance is a strategy designed to detect cancer, not to prevent cancer. Over the past decade, research efforts have been directed toward endoscopic mucosal ablation to eliminate the risk of cancer and allow regeneration of squamous epithelium following pharmacological or surgical control of reflux. ${ }^{26}$ The clinical value of endoscopic ablation of nondysplastic BE combined with acid suppression therapy or antireflux surgery remains controversial.

APC, at a power output of 60-90 Watts, allows a safe ablation of BE and LGD with a high success rate. In our series including 94 patients with nondysplastic $\mathrm{BE}$, the eradication rate was $72.3 \%$ with an average of three treatment sessions. Of the 68 eradicated patients, 27 underwent fundoplication and 41 were treated with proton pump inhibitors; the eradication rate was $89.4 \%$ and $87.5 \%$, respectively, with a mean follow-up of 26 months. ${ }^{27}$ Bright et al. recently published the results of a randomized trial of APC versus endoscopic surveillance for BE after antireflux surgery. Regression of BE after fundoplication was more likely, and greater in extent, in patients who underwent prior ablation with APC. Moreover, in most patients treated with APC, the newly developed squamous epithelium remained stable at up to five-years follow-up. The only case of progression to HGD occurred in a patient who was not treated with APC before surgery. ${ }^{28}$

\section{Optimizing techniques for RFA to treat BE}

Richard I. Rothstein, MD

richard.i.rothstein@hitchcock.org

Attention to radiofrequency device evolution and procedural techniques, along with individualized patient factors, will permit optimization of outcomes from this innovative and beneficial therapy for BE. RFA is optimal for treating flat, nonnodular $B E$. Individuals with nodular disease may be treated with RFA following the removal of the nodular segments with endoscopic mucosal resection (EMR). A delay of six to eight weeks between the time of EMR to the performance of RFA of Barrett's tissue should provide adequate time for healing of the resected mucosa and is necessary for the best treatment outcomes. RFA is a safe and effective treatment for dysplastic Barrett's as well as intestinal metaplasia with foci of intramucosal carcinoma (staged by endoscopic ultrasound and EMR). Selected nondysplastic Barrett's patients can be safely and effectively treated with RFA and it can be cost-effective for the management of that condition compared to the surveillance strategies in current practice.

The performance of RFA involves the use of specialized devices and a sophisticated control unit (Halo 360 and 90, Barrx Medical). With the Halo RFA system, thermal energy is precisely delivered to the treated tissue to a depth of 500-1000 $\mu \mathrm{m}$, to remove the mucosal layer and limit damage to the deeper submucosal layer. That effectively reduces the problem of stricture formation, which is a major problem with other ablation treatments such as wide-field EMR and PDT.

The Halo 360 system uses a sizing balloon catheter and variable-sized electrode treatment balloons. The procedure is done following a standard upper endoscopic exam during which the landmarks 
are recorded for the upper border of the Barrett's segment and the top of the gastric folds as well as the geographic pattern of the intestinal metaplasia. The Barrett's segment is cleaned with a spray of dilute $n$-acetylcysteine to remove surface mucous and a guidewire is left in the distal stomach as the gastroscope is removed. Over this guidewire, the sizing balloon is passed and localized $12 \mathrm{~cm}$ proximal to the top of the gastric folds (placed even higher if needed to be above the proximal extent of the Barrett's for ultra-long segment disease). Stepwise inflation and deflation of the balloon as it is moved centimeter-by-centimeter towards the cardia allows the determination (shown on the digital monitor of the control unit) of the size of the esophageal lumen, which is used to determine the effective treatment balloon size. In the situation of multiple balloon sizes recorded along the length of the esophagus, the recommendation is to use the smallest treatment balloon size recommended. However, in the situation of smaller proximal lumen and a consistently larger distal lumen, as may be seen in longer-segment disease, it is appropriate to use a smaller treatment balloon proximally and the larger size distally. In all situations, it is important to view that the selected treatment balloon size permits effective contact of its electrodes to the esophageal wall.

After the sizing steps have been completed, the sizing balloon is removed and the selected treatment balloon is introduced over the guidewire. The gastroscope is introduced alongside the Halo 360 catheter since the RFA treatment is done under direct vision. The proximal part of the electrode segment of the treatment balloon is best placed 1 $\mathrm{cm}$ proximal to the top of the Barrett's segment. For Barrett's with dysplasia, the setting for ablation is typically set on the unit at 12 joules $/ \mathrm{cm}^{2}$, whereas for nondysplastic it is recommended to be 10 joules $/ \mathrm{cm}^{2}$. The Barrett's segment is treated proximal to distal with a $5 \mathrm{~mm}$ to $1 \mathrm{~cm}$ overlap in order to obtain uniform treatment without "skip" areas. Once treated, the endoscope and the balloon with guidewire are all removed from the patient. A soft sort distal cap is placed on the gastroscope, which is reintroduced and used to scrape away all of the treated sloughing mucosa. This step is very important to optimize the ablation outcome, and enough time should be spent to ensure adequate cleaning of the treated segment. The guidewire is replaced

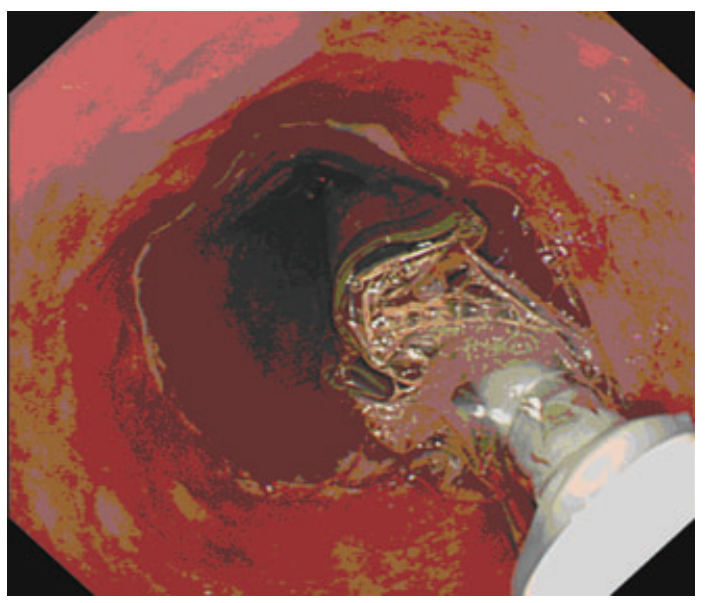

Figure 4. Halo 360 postablation view.

into the stomach and the endoscope removed. The treatment technique of balloon passage and use is repeated as described, with all of the Barrett's segment treated a second time. Following removal of the endoscope, balloon, and guidewire, the patient is recovered, and instructed to continue on bid proton pump inhibitors and to consume a liquid diet for two days and then a soft diet for three to five days before resuming a regular diet. Mild analgesics may be needed, and patients should be given instructions and education about the typical sensations to expect following the RFA treatment (Fig. 4).

In follow-up RFA sessions where there may be residual small areas of Barrett's remaining after the circumferential balloon treatment, the Halo 90 system may be employed. This endoscope-mounted system, with an electrode pattern of $13 \times 20 \mathrm{~mm}$, can also be used for primary therapy of short segment disease. After a standard upper endoscopy, the Halo 90 device is mounted on the endoscope which is reintroduced and used in a patchwork fashion with $3 \mathrm{~mm}$ overlap onto treated segments to treat all areas of intestinal metaplasia. In contrast to the single application of thermal energy with the circumferential balloon, followed by cleaning of the segment and then retreatment, with the Halo 90 the Barrett's mucosa is treated twice, then scraped clean, and then retreated. Instead of a cap, the mounted RFA device can be used for removal of the sloughed tissue. When treating short segment disease, the device is placed to straddle the top of the gastric folds, and the treatments done by rotating the scope to permit an overlap of a few millimeters and moving 
around the gastroesophageal junction, usually taking six treatment sites to cover the circumference. Posttreatment recommendations are as for the circumferential treatment described.

As for all new advanced endoscopic procedures, adequate training and mentoring are essential to optimize the clinical outcomes and ensure the best safety for RFA of Barrett's. For references to this section, see Refs 6, 21, 29, 30 and 31.

\section{Ablation of nondysplastic Barrett's epithelium: a surgeon's perspective}

\author{
Vic Velanovich \\ vvelano1@hfhs.org
}

Ablation of $\mathrm{BE}$ has been shown to be an effective means of eliminating Barrett's epithelium ${ }^{32}$ and to decrease the incidence of EAC in patients with HGD. ${ }^{6}$ Nevertheless, controversy exists as to whether or not ablating nondysplastic Barrett's epithelium should be done. ${ }^{22}$ This paper provides the surgeon's perspective on this issue.

The concept of eliminating cancer-prone epithelium is not new to surgeons. There are several disease processes which have had operations designed specifically to reduce cancer risk (Table 5). Therefore, it is not a conceptual leap for the surgeon to apply ablative techniques to Barrett's epithelium to reduce cancer risk.

Although the risk of EAC in BE is low, prevention is still worthwhile due to the serious consequences of developing adenocarcinoma. EAC is primarily treated with esophagectomy. Esophagectomy carries significant and not easily ignored immediate

Table 5. Diseases with cancer-prone epithelium and operations designed to reduce cancer risk

\begin{tabular}{lc}
\hline Disease & Operation \\
\hline Colonic adenoma & Colonoscopy polypectomy \\
Ulcerative colitis & Total proctocolectomy \\
Familial adenomatous & Total colectomy with \\
polyposis-colon & rectal mucosal stripping \\
Familial adenomatous & Pancreas-sparing \\
polyposis-duodenum & duodenectomy \\
Hereditary medullary & Total thyroidectomy \\
carcinoma & \\
BRCA mutations-breast & Bilateral total mastectomy \\
cancer & \\
\hline
\end{tabular}

and long-term adverse events. In the short-term, even in the best of hands, the morbidity rate is over $50 \%$, with a measurable mortality rate. In general practice, the mortality rate can be as high as $10 \%$. Stricture at the anastomosis can occur in 25$50 \%$ of patients. This not only leads to dysphagia, but may require multiple dilations. Long-term, even with curative surgery, the quality of life effects of an esophagectomy are substantial. Djarv et al. ${ }^{33}$ have shown that three years after curative esophagectomy for esophageal cancer, patients have lower quality of life score compared to population norms in several physical functioning domains as well as several constitutional symptoms. Therefore, prevention of an esophagectomy is almost as worthwhile goal as the prevention of adenocarcinoma. In many respects, ablation of Barrett's epithelium can be considered a means of preventing esophagectomy.

$\mathrm{BE}$ can recur after ablation. This is prevented by a strict regimen of acid-suppression therapy. However, the development of Barrett's is promoted most aggressively by the combination of acid and bile reflux. Although proton pump inhibitor therapy is effective in reducing acid reflux, it does nothing for bile reflux. We have previously shown that the addition of an antireflux operation, most typically a laparoscopic Nissen fundoplication, reduces the rate of recurrent or persistent BE. ${ }^{34}$ Clearly, a randomized trial is needed to confirm these findings.

\section{RFA for nondysplastic BE: the gastroenterologist's perspective}

Srinadh Komanduri

koman1973@gmail.com

Perhaps the most intriguing aspect of endotherapy for $\mathrm{BE}$ is its application in nondysplastic disease. Although offering therapy for patients with dysplastic $\mathrm{BE}$ is a cost-effective strategy, the same is not so evident for nondysplastic disease. However, treating only patients with dysplasia will likely limit our ability to reach the ultimate goal of eradication of EAC.

RFA offers an effective, safe, and durable treatment for patients with BE. Most recently, five years data from the AIM II trial demonstrated 92\% complete eradication of BE (CR-IM). This is extremely exciting data and encourages to expand our indications for endotherapy in BE. Although RFA 
in nondysplastic BE appears to be cost-effective if surveillance can be discontinued, it remains to be seen where this fits in the management paradigm when surveillance is continued.

Currently, there is insufficient data to recommend cessation of surveillance after endotherapy despite achieving CR-IM. A potential solution is to riskstratify our patients with $\mathrm{BE}$ for consideration for RFA. Although many biomarkers are under investigation, we are still far from a simple blood test to define risk in nondysplastic BE. That being said, we currently have a safe therapy that can eliminate not only disease, but any of these molecular changes that may drive stem cell differentiation. In addition, we must consider quality of life (QOL) in this cohort. It is considered standard of care to eliminate all precancerous lesions in the body (e.g., colon polyps, breast lumps, and actinic keratosis). This paradigm creates a dilemma when we tell our patients that we will not remove their precancerous BE. It is difficult for patients to consider the small rate of progression to cancer in these circumstances. Therefore, we must consider how this would affect a patient's quality of life when making treatment decisions. Previous data have shown incremental improvement in QOL with successful eradication of BE. Ultimately, it would be ideal to have data that demonstrates a true cancer reduction with implementation of RFA as a treatment strategy in nondysplastic BE. However, given the progression rate, this will likely not be feasible any time soon. Despite the lack of this data, the durability of RFA and a recent meta-analysis clearly suggests that implementation of RFA would decrease the incidence of EAC.
Currently, RFA should be considered in select patients who fully understand the limitations of currently available data. These may include patients who are young (age $<50$ ), those with long segment disease, and patients with family history of BE-dysplasia or EAC. Regardless of this choice, it is imperative to have an extensive discussion with these patients and discuss the current data to allow them to make an educated decision about their disease. [For references to this section, see Refs 35-38]

\section{What is the mechanism of regression of Barrett's esophagus after BWR?}

Edgardo Diaz-Cervantes, A. De-la-Torre-Bravo, S. Sobrino-Cossio, E. Torres-Durazo, O. Martínez-Carrillo, and J. Gamboa-Robles edgardoscopio@hotmail.com

Because Barrett's epithelium has a thickness of only 0.5 to $0.6 \mathrm{~mm},{ }^{39}$ banding might be expected to result in its complete eradication. Indeed, an event in our endoscopy unit revealed an ablative technique for Barrett's esophagus (BE) that could fulfill a number of the ideal criteria for an ablative alternative. During an endoscopic variceal ligation in a patient who had bleeding esophageal varices, we placed a band on a varix that had an overlying tongue of BE. Proton pump inhibitors were prescribed. To our surprise the BE was no longer visible on a follow-up endoscopic examination one month later; this observation suggested that banding had eradicated the BE. $^{18}$

\begin{tabular}{|c|c|c|c|c|c|c|}
\hline \multicolumn{7}{|c|}{ ALL METHODS OF ABLATION } \\
\hline METHOD & CR-IM & $\begin{array}{l}\text { SCAR } \\
\text { THICKNESS }\end{array}$ & STENOSIS & $\begin{array}{c}\text { PERFORATION } \\
x\end{array}$ & $\begin{array}{l}\text { BLEEDING } \\
z\end{array}$ & $\begin{array}{c}\text { BURIED } \\
\text { BARRETT'S } \\
x\end{array}$ \\
\hline CRYO & 21 & & & & & $0-18$ \\
\hline MPEC & 67 & & 1 & & $0-14$ & \\
\hline APC & $55 \cdot 76$ & & $0-15$ & $0-3$ & $0-4$ & \\
\hline RFA & 54.89 & & & 0.03 & -1 & 0.6 \\
\hline PDT & 52 & & $23-35$ & $0-1$ & $0-14$ & $6-60$ \\
\hline BWR & $98 \%$ & THIN & 0 & 0 & 0 & 0.8 \\
\hline MBL-EMR & & CROSs & $10-70$ & $0-9$ & $0-10$ & $?$ \\
\hline
\end{tabular}

Figure 5. Methods of ablation. 


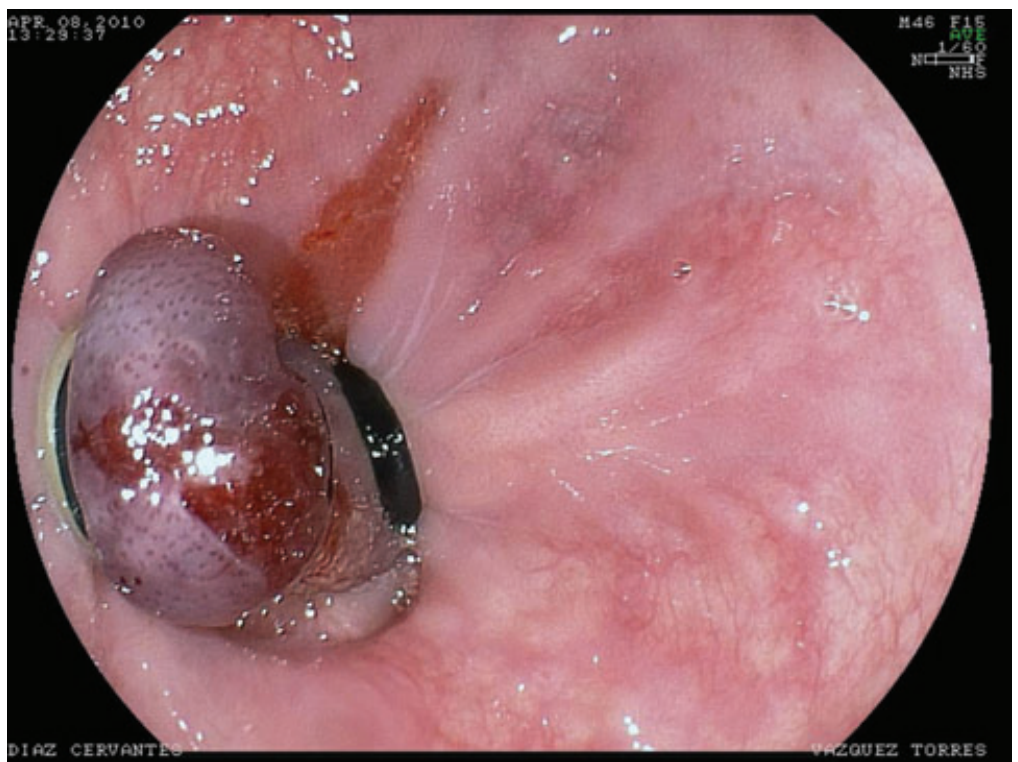

Figure 6. Banding without-resection.

Banding without-resection (BWR) is a novel method of ablation for BE, consisting on the creation of a pseudopolyp by using a multib and ligation device. Its main feature resides in a nonresection technique of the created pseudo-polyp, leaving it just ligated until it spontaneously sloughs off (Fig. 5), leaving a small ulceration that heals with neosquamous epithelium.

\section{Mechanism of regression}

1. Ischemia: the band obliterates the blood flow, and 5 to 10 days later the ischemic tissue sloughs off, leaving a small ulceration that heals with the replacement of neosquamous mucosa when acid reflux is controlled by the administration of PPI.

2. The defect of the banded mucosa is replaced by surrounding mucosa. This means that the smaller mucosal defect leads to smaller residual ulcer, resulting into a small, thin, and superficial scar.

2. By BWR, we don't snare, cut, heat; we neither infiltrate nor inject; we just apply suction, band, wait, and follow-up... so simple... so "easy" on the esophagus.

One drawback of BWR is that there is no tissue for histological evaluation; but this is a drawback shared by all ablative in situ-destroying procedures for BE, except for EMR (Fig. 6).

\section{Depth of ablation}

Because BWR depends on the creation of a pseudopolyp, and the same principle rules for endoscopic mucosal resection (EMR): then, both procedures may have similar depth of ablation. Endoscopic banding has an excellent track record of safety, even in high risk patients with cirrhosis. There are many substantial advantages on BWR when compared to resection, such as:

1. Banding without-resection is per se preventive for bleeding and perforation.

2. No need for submucosal injection before banding.

3. The risk of stricture after banding withoutresection is minimal or absent. As a result of BWR we obtain only a thin, small and superficial scar, instead of big mucosal defects with long and gross scars that are usually obtained after resection.

\section{Conflicts of interest}

The authors declare no conflicts of interest.

\section{References}

1. Larghi, A. et al. 2005. EUS followed by EMR for staging of high-grade dysplasia and early cancer in Barrett's esophagus. Gastrointest. Endosc. 62: 16.

2. Prasad, G.A. et al. 2007. Long-term survival following endoscopic and surgical treatment of high-grade 
dysplasia in Barrett's esophagus. Gastroenterology 132: 1226-1233.

3. Pech, O. et al. 2008. Long-term results and risk factor analysis for recurrence after curative endoscopic therapy in 349 patients with high grade intraepithelial neoplasia and mucosal adenocarcinoma in Barrett's Oesophagus. Gut 57: 1200.

4. Wolfsen, D. 2009. Endoscopic ablation therapy: imaging and advanced technology in action. Gastroenterology 137: 1225.

5. Overholt, B.F. et al. 1995. Barrett's esophagus: photodynamic therapy for ablation of dysplasia, reduction of specialized mucosa and treatment of superficial esophageal cancer. Gastrointest. Endosc. 42: 64-69.

6. Shaheen, N.J. et al. 2009. Radiofrequency ablation in Barrett's esophagus with dysplasia. N. Engl. J. Med. 360: 22772288.

7. Pouw, R.E. et al. 2010. Safety outcomes of Balloon-based RFA after focal endoscopic resection of early Barrett's neoplasia in 118 patients: results of an ongoing European multicenter study. Gastrointest. Endosc. 71: AB126.

8. Schiefke, I. et al. 2005. Long term failure of endoscopic gastroplication (EndoCinch). Gut 54: 752-758.

9. Pleskow, D. et al. 2004. Endoscopic full-thickness plication for the treatment of GERD: a multicenter trial. Gastrointest. Endosc. 59: 163-171.

10. CadiEre, G.B. et al. 2008. Endoluminal fundoplication by a transoral device for the treatment of GERD: a feasibility study. Surg. Endosc. 22: 333-342.

11. CadiEre, G.B. et al. 2008. Antireflux transoral incisionless fundoplication using EsophyX: 12-month results of a prospective multicenter study. World J. Surg. 32: 1676-1788.

12. Garewal, H., L. Ramsey, P. Sharma, et al. 1999. Biomarker studies in reversed Barrett's esophagus. Am. J. Gastroenterol. 94: 2829-2833.

13. Krishnadath, K.K., K.K. Wang, K. Taniguchi, et al. 2000. Persistent genetic abnormalities in Barrett's esophagus after photodynamic therapy. Gastroenterology 119: 624-630.

14. Hage, M., P.D. Siersema, K.J. Vissers, et al. 2005. Molecular evaluation of ablative therapy of Barrett's oesophagus. $J$. Pathol. 205: 57-64.

15. Prasad, G.A., K.K. Wang, K.C. Halling, et al. 2008. Correlation of histology with biomarker status after photodynamic therapy in Barrett esophagus. Cancer 113: 470-476.

16. Dvorak, K., L. Ramsey, C.M. Payne, et al. 2006. Abnormal expression of biomarkers in incompletely ablated Barrett's esophagus. Ann. Surg. 244: 1031-1036.

17. Urosevic, P. \& G.K. Kiroff. 2002. Ablation of Barrett's epithelium: the promise and the problems diseases of the esophagus 15: $30-38$.

18. Diaz-Cervantes, E., A. De-la-Torre-Bravo, S.J. Spechler, et al. 2007. Banding without resection(endoscopic mucosal ligation) as a novel approach for the ablation of short-segment Barrett's epithelium: results of a pilot study. Am. J. Gastroenterol. 102: 1640-1645.

19. Saadi, A., N.B. Shannon, P. Lao-Sirieix, et al. 2010. Stromal genes discriminate preinvasive from invasive disease, predict outcome, and highlight inflammatory pathways in digestive cancers. PNAS 107: 2177-2182.
20. Fleischer, D.E., B.F. Overholt, V.K. Sharma, et al. 2008. Endoscopic ablation of Barrett's esophagus: a multicenter study with 2.5-year follow-up. Gastrointest. Endosc. 68: 867876.

21. Fleischer, D.E., R. Odze, B.F. Overholt, et al. 2010. The case for endoscopic treatment of non-dysplastic and lowgrade dysplastic Barrett's esophagus. Dig. Dis. Sci. 55: 1918-1931.

22. Fisher, R.S., M.Q. Bromer, R.M. Thomas, et al. 2003. Predictors of recurrent specialized intestinal metaplasia after complete laser ablation. Am. J. Gastroenterol. 98: 19451951.

23. Spechler, S.J. 2002. Barrett's esophagus. N. Engl. J. Med. 346: 836-842.

24. Romagnoli S., M. Roncalli, D. Graziani, et al. 2001. Molecular alterations of Barrett's esophagus on microdissected endoscopic biopsies. Lab. Invest. 81: 241-247.

25. Incarbone R., L. Bonavina, G. Saino, et al. 2002. Outcome of esophageal adenocarcinoma detected during endoscopic biopsy surveillance for Barrett's esophagus. Surg. Endosc. 16: 263-266.

26. Bonavina L., C. Ceriani, A. Carazzone, et al. 1999. Endoscopic laser ablation of nondysplastic Barrett's epithelium: is it worthwhile? J. Gastrointest. Surg. 3: 194-199.

27. Pagani M., P. Granelli, B. Chella, et al. 2003. Barrett's esophagus: combined treatment using argon plasma coagulation and laparoscopic antireflux surgery. Dis. Esoph. 16: 279283.

28. Bright T., D.I. Watson, W. Tam, et al. 2007. Randomized trial of argon plasma coagulation versus endoscopic surveillance for Barrett's esophagus after anti-reflux surgery: late results. Ann. Surg. 246: 1016-1020.

29. Lyday W.D., F.S. Corbett, D.A. Kuperman, et al. 2010. Radiofrequency ablation of Barrett's esophagus: outcomes of 429 patients from a multicenter community practice registry. Endoscopy 42: 272-278.

30. Fleischer, D.E., B.F. Overholt, V.K. Sharma, et al. 2010. Endoscopic radiofrequency ablation for Barrett's esophagus: 5 -year outcomes from a prospective multicenter trial. Endoscopy 42: 781-789.

31. Vassiliou, M.C., D. von Renteln, D.C. Wiener, et al. 2010. Treatment of ultralong segment Barrett's using focal and balloon-based radiofrequency ablation. Surg. Endosc. 24: 786-791.

32. Velanovich, V. 2009. Endoscopic endoluminal radiofrequency ablation of Barrett's esophagus: initial results and lessons learned. Surg. Endosc. 23: 2175-2180.

33. Djarv, T., J. Lagergren, J.M. Blazeby \& P. Lagergren. 2008. Long-term health-related quality of life following surgery for oesphageal cancer. Br. J. Surg. 95: 1121-1126.

34. O’Connell, K. \& V. Velanovich. 2010. Effects of Nissen fundoplication on endoscopic endoluminal radiofrequency ablation of Barrett's esophagus. Surg. Endosc.

35. Wani, S. et al. 2009. Esophageal adenocarcinoma in Barrett's esophagus after endoscopic ablative therapy: a metaanalysis and systematic review. Am. J. Gastroenterol. 104: 502-513 [Review].

36. Fleischer, D. et al. 2010. AIM II: 5 year durability data. DDW Plenary. 
37. Wang, J.S., M. Guo, E.A. Montgomery, et al. 2009. DNA promoter hypermethylation of p16 and APC predicts neoplastic progression in Barrett's esophagus. Am. J. Gastroenterol. 104: 2153-2160 [Epub 2009 Jul 7].

38. Shaheen, N.J., A.F. Peery, R.H. Hawes, et al. 2010. AIM Dysplasia Trial Investigators. Quality of life following radiofre- quency ablation of dysplastic Barrett's esophagus. Endoscopy 42s: 790-799 [Epub 2010 Sep 30].

39. Ackroyd, R., N.J. Brown, T.J. Stephenson, C.J. Stoddard, \& M.W.R. Reed. 1999. Ablation therapy for Barrett oesophagus: what depth of tissue destruction is needed? J. Clin. Pathol. 52: 509-512. 